\title{
REAL-TIME ACQUISITION AND ANALYSIS METHOD AND SYSTEM OF SECURITY HIDDEN DANGER INFORMATION IN INTELLIGENT SCENIC SPOTS UNDER THE ENVIRONMENT OF INTERNET OF THINGS
}

\author{
Baishou $\mathrm{Li}^{1,2}$, Yuehui Xie ${ }^{1, *}$ \\ ${ }^{1}$ College of Geomatics and Geoinformation, Guilin University of Technology, Guilin, Guangxi, China - (xyh_qz, \\ lbszhb)@163.com \\ ${ }^{2}$ Guangxi Key Laboratory of Spatial Information and Geomatics, Guilin, Guangxi, China - lbszhb@ 163. com
}

KEY WORDS: Internet of Things, Rainfall-Sensor, Water Depth Sensor, Safety Hazard Information, Acquisition and Analysis, Real-time System

\begin{abstract}
:
Aiming at the problem that the existing methods of collecting and processing information of potential location safety hazards in scenic spots can not identify and analyze real-time potential hazards quickly and accurately, a real-time method and system of collecting and analyzing information of potential location safety hazards for tourists in intelligent scenic spots under the environment of Internet of Things is proposed.The set of method and system includes: collecting geographical coordinate information of potential safety hazards threatening visitor through the handheld terminal, and connecting the collected data and the spatial analysis request to the GIS spatial analysis server through the Web server in real time, so as to realize the buffer analysis considering the gradient; based on the knowledge base of secure locations in scenic spots and internet of things equipment, such as rainfall-sensor and water depth sensors, the radius of hidden danger buffer and parameter information of warning level are calculated in real time, and the knowledge formed in the analysis process is saved. By comparing and analyzing the historical information of Location Safety Knowledge Base, the temporal and spatial variation rules of newly added hidden danger features are discovered to determine the optimal value of hidden danger parameters. Then, through the geographic information system network service software, the new hidden danger buffer element layer is sent to the handheld terminal in real time, so as to avoid the impact of unsafe tourist caused by the untimely updating of hidden danger information in scenic spots, and improve the efficiency of safe production in scenic spots.
\end{abstract}

\footnotetext{
* Corresponding author

E-mail addresses: xyh_qz@163.com
}

\section{INTRODUCTION}

With the development and application of new technologies such as mobile communication, Internet of Things and cloud computing, the concepts of Smart Earth, Smart City and Smart Scenic Spot(Chen et al.,2012) have been born since 2008. The concept of intelligent scenic spot has added new connotation to the informationization construction of scenic $\operatorname{spot}($ Shao et al.,2010)(Dang et al.,2011). It is a leap development on the basis of digital scenic spot and represents the new direction of informationization construction of scenic spot(Dang et al.,2011).In academia, the field of "intelligent scenic spots" has increasingly become the focus of scholars' attention.Documents (Hanlin,2014)(Wang et al.,2015)(Huang,2015) respectively studied the construction of intelligent scenic spots, and achieved some research results. However, there are few studies on the safety supervision and intelligent early warning of "smart scenic spots".In recent years, only representative achievements have been reported: Wang Qiuju's framework research and Empirical Analysis on the early warning of tourist flow in scenic spots based on network attention(Wang et al.,2014); Youweibin's construction and application of the early warning system of ecological security in the world's dual heritage sites(You et al.,2014).This shows that the domestic research on intelligent early warning of scenic spots is still a weak link, and there is still much room for improvement on the basis of existing research results.Safety is the basis of tourism development. It is not only the guarantee for the smooth development of tourism activities, but also the prerequisite for tourism development (Hua et al.,2009). The safety of tourists is very important for scenic spots. The natural scenery, which is emphasized by the words "strange" and "dangerous", often hides many hidden dangers of nature and man-made that threaten tourists. With the vigorous development of the intelligent tourism industry and the increasing number of scenic spots, the security problems of scenic spots are increasing day by day.Many scenic spots have complex topography and landforms, and there are many hidden dangerous and threatening areas with point, line and surface shapes, which are randomly distributed in the scenic area, which poses a great threat to the location safety of tourists in scenic spots.The influence range of hidden dangers in scenic spots is different according to the types of hidden dangers, and it changes with the month or precipitation. For example, the boundary of natural lakes in scenic spots changes with the precipitation, and the range of activities of wild monkeys also changes regularly or periodically with the season, month and tourist activity area. A more scientific and effective method of spatial information acquisition and processing analysis is needed to obtain the influence scope of similar hidden danger areas.

At present, the method of collecting and processing hidden danger information of scenic spots still remains in manual record and off-line manual collection of spreadsheet storage system(Wang et al.,2013). However, the existing schemes can not quickly and accurately identify hidden danger in real-time and fast spatial analysis. Therefore, it has an important impact on the efficiency of real-time acquisition of potential location 
security hazards.In many scenic spots, only when the visitors have a location safety accident, the hidden dangers of scenic spots are investigated safely, which can not make up for the loss of sheep and make up for them afterwards. Moreover, there is a lack of convenient technology for real-time collection, storage and updating of hidden dangers in scenic spots to meet the needs of real-time collection and dynamic analysis of spatial and temporal data of hidden dangers in scenic spots.

In view of the existing problems, this paper proposes a real-time collection and analysis method and system of potential location safety hazard information for intelligent scenic spots under the environment of the Internet of Things(Jia,2010).Considering not only the change of slope in scenic spots, but also the hidden danger buffer zone of topographic slope is analyzed with the collected data of point hidden danger, line hidden danger and surface hidden danger. The real-time and spatial analysis technology of GIS is used to plot the potential hidden danger threat range in scenic spots.It can collect and analyze the existing hidden danger data in real-time space to meet the requirement of real-time updating and storage of intelligent scenic spots, and solve the key problems of inaccurate updating of hidden danger information and inaccurate location security information of tourists in scenic spots for early warning of hidden danger.

\section{REAL-TIME ACQUISITION AND ANALYSIS METHOD OF HIDDEN DANGER INFORMATION OF POSITION SAFETY}

\subsection{Procedure of Information Acquisition Method for Hidden Hazards of Location Safety}

The flow chart of a real-time acquisition and analysis method for potential location safety hazard information of tourists in intelligent scenic spots under the environment of Internet of Things is shown in Figure 1.

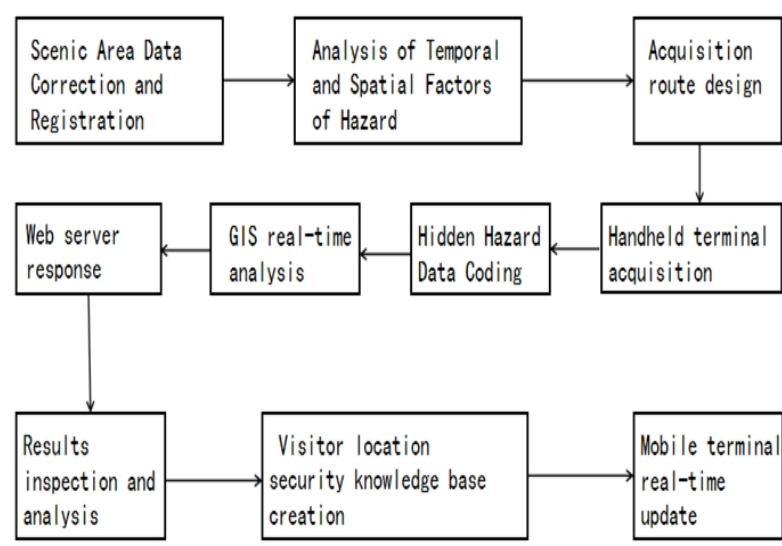

Figure 1. Flow chart of real-time acquisition and analysis method for potential position safety hazard information

\subsection{Data preprocessing}

2.2.1 Scenic Area Data Correction and Registration: LocaSpaceViewer software is used to acquire high-resolution satellite image data (spatial resolution is higher than $1 \mathrm{~m}$ ) in recent years, and several points coordinates with uniform distribution which have not changed in recent years are selected as high-resolution image geographic correction and registration control points. In order to ensure the spatial consistency between image data and field acquisition data, all image data and potential safety hazard location data are projected into a unified projection coordinate system.

The high resolution digital elevation model (DEM scale greater than 1:1000) of scenic spot is obtained by using UAV stereo imaging equipment.

\subsubsection{Temporal and Spatial Factors Analysis of Hidden}

Hazards: Investigate the natural hazards and tourist route factors, including month and precipitation, water depth caused by tourism insecurity factors, analyze and summarize the different laws of each month of each year, as well as study and analyze the relationship between the threat of hidden hazards and tourist flow of scenic area precipitation or tourist routes in different months.

Statistical analysis was made on the data of tourists' flow into the scenic area over the years and every month, the density of tourists' activity area monitored by video, and the flow of tourists along tourist routes. This paper analyses the regularity of tourist flow in each month and the relationship with weather factors in that month, and comprehensively analyses the impact scope of disasters caused by weather conditions, which threatens public facilities such as tourist trails, roads and bridges in scenic spots.For example, flood, collapse, landslide, debris flow, wild monkey herd activities and other factors caused by the security risks of buffer radius updating and frequent impact on tourist safety hot spots.

Combining with the historical image of the scenic area, the UAV image is used for topographic survey and geomorphological analysis of the scenic area. The hidden danger points and areas are selected through geomorphological analysis, and the visible hidden danger waters are extracted from high-resolution satellite images.

\subsubsection{Route Design and Data Acquisition and Coding:} Combining the image data to determine the hidden dangers of safety points, lines and areas that have been found or potential, and considering the topography, precipitation, location distribution of tourists and location flow data statistics, the range of hidden danger areas that change dynamically with the month and the optimized geographical location of hidden danger areas which are closer to the actual situation are obtained.According to the distribution of potential safety hazards, the location of the scenic spot is selected as the analog acquisition and calibration field, and the route of information acquisition of potential safety hazards for tourists is determined and marked in the map layer.

According to the established information acquisition route, the handheld terminal is used to collect the identified or potential dangerous points in the system. The coordinate information collected includes coordinate $(x, y)$, and the corresponding acquisition time and hidden danger types of each acquisition point data are recorded. The hidden danger of a line can be determined by several nodes, and the accuracy of the hidden danger position can be determined by collecting appropriate coordinates at the corner point. At the corner point of a polygon, many coordinate points can be collected to ensure the accuracy of the polygon.

The hidden danger information of scenic spots is divided into three types: point hidden danger, line hidden danger and surface hidden danger, as shown in Figure 2. Point hidden danger mostly refers to the location of rocks in Shishan, the location of steep slopes, the location of unopened cave entrance, etc.Line 
hidden danger mainly refers to the section where there is no barrier or the gap between barriers is too wide, where rivers, tidal boundaries and bridges are easy to fall rocks, forming debris flow and landslides; surface hidden danger mainly refers to the polygonal waters without barriers, the active areas of wild monkeys, etc. Point, line and surface of hidden dangers also include those not open in the scenic area, but visitors can venture into point, line and surface hidden dangers. The coding content includes hidden danger information type, 01 representative point hidden danger, 02 representative line hidden danger, 03 representative surface hidden danger data, and data acquisition time $\mathrm{T} 1 \sim \mathrm{Tn}$ and minimum radius $r_{\mathrm{min}}$ of hidden danger buffer are necessary in coding. Point hidden danger, line hidden danger and surface hidden danger are coded and stored according to code.

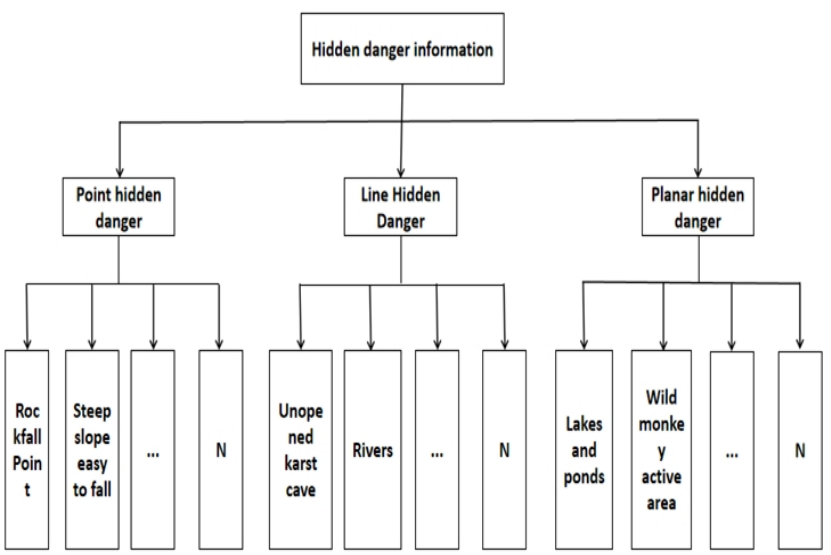

Figure 2. Information Division Map of Security Hidden Dangers in Scenic Spots

\subsection{Realization of Real-time Data Acquisition and Analysis} Method for Potential Position Security Hazards

2.3.1 Real-time acquisition of hidden danger data in the Internet of Things: Based on rainfall and water depth sensors, real-time water depth information of lakes, ponds and rivers in scenic spots or the prominent points of the periphery of a lake without barriers or a tidal bore with too wide gap between barriers can be obtained.By changing the boundary of the lake polygon and the hidden dangerous area of wild monkeys dynamically through water depth and month parameters, the radius $r$ of the buffer area in the neighborhood is increased: $\mathrm{r}=r_{\min }+\Delta r$, where $\mathrm{r}_{\min }$ is the minimum radius of the buffer area, and the radius increment is recorded as $\Delta \mathrm{r}$, and recorded in the knowledge base of the location safety of the scenic spot.The hidden dangers of wild monkey herds are buffered by month, and the buffer areas of rivers without barriers and tidal bores are built by hour, so that the neighborhood radius of the buffer zone can be obtained dynamically with time.

Monthly parameters determine the buffer radius $r$ of wild monkeys. The buffer radius collected in different months is recorded separately. Rivers without barriers and tidal surges are calculated according to the increment of neighborhood radius of buffer zone established by hour: $\Delta \mathrm{r}=a \times \Delta h+b$, where $\Delta \mathrm{h}$ is increment of hour water depth; $a$ and $b$ are undetermined coefficients of equation, which are calculated by historical water depth records and historical farthest points of roadways, roads and bridges crossed by tidal surges or water bodies.
The daily rainfall $d r$ recorded in the database determines the hidden danger grade $\mathrm{D}$ of the collapse and fall points on that day. The higher the daily rainfall, the higher the warning level of hidden dangers. The gradation of $\mathrm{D}$ is determined by the piecewise function, and the piecewise threshold is determined by the critical rainfall threshold of hidden danger occurrence in the location safety knowledge base.

\subsubsection{Real-time Analysis of Points, Lines and Surfaces of} Hidden Dangerous Data Based on GIS: Through the GIS spatial analysis server, the data of point hidden danger, line hidden danger and surface hidden danger are analyzed in buffer area.The analysis of hidden danger buffer zone in scenic spots aims at receiving or displaying early warning information through intelligent information platform of scenic spots, electronic screen, scenic area broadcasting and other Internet of Things information devices in the process of approaching hidden danger within a specific distance of certain points, lines or areas in scenic spots, so as to avoid the occurrence of unsafe events of tourist location.For a given geometric object $\mathrm{A}$, the buffer of $\mathrm{A}$ is defined as $\mathrm{P}=\{x \mid d(x, A) \leq r\}$ ( $\mathrm{x}$ is the buffer boundary, $\mathrm{d}$ is the Euclidean distance formula), and the neighborhood threat radius of different kinds of potential security hazards is derived from the scenic spot location security knowledge base $r$.

The buffer area of the collected hidden danger data is analyzed. See Figure 3. The buffer radius calculated in the twodimensional map is :

$$
r=d \times \cos (\theta)
$$

where $\quad r=$ a two-dimensional projection radius $\mathrm{d}=\mathrm{a}$ three-dimensional distance in the field $\theta=$ a slope angle

The basic calculation and analysis methods of point hidden danger buffer are as follows: the buffer is set as a plane geometric area with $\mathrm{P}$ as the center and $\mathrm{r}$ as the radius. Taking the collapse rock buffer as an example, referring to Figure 3, the calculation results of buffer radius at different slope positions are different.The slope map is derived from DEM by using GIS raster terrain analysis tool, and the slope angle is changed from radial unit to angular unit. The falling stone point $\mathrm{P}$ is located beside the road and the walking path in the scenic area, and the influence range is closely related to the terrain slope. See Figure 4 , the distance between the buffer boundary and the hidden dangerous point $\mathrm{P}$ changes with the change of terrain slope. The larger the slope is $(\theta 1 \geq \theta 2)$, the smaller the radius of the projection buffer is $(\mathrm{R} 1 \leq \mathrm{R} 2)$. When $\theta 1=\theta 2$, the buffer radius $\mathrm{R} 1=\mathrm{R} 2$. $\mathrm{r}$ value, $\mathrm{r}_{\min } \leq r \leq r_{\max }$.

Line hidden danger buffer zone uses one side of the axis as a buffer zone in a certain range. In visible waters with potential safety hazards, a multi-point broken line is used to make a buffer zone at a certain distance outside the broken line. The basic calculation and analysis methods are as follows: in the two-dimensional map, the buffer radius $\mathrm{R}$ is calculated according to the specific formula (1), then the buffer zone is set to be centered on the L-line (river center line).A plane geometric region with a radius of $r$. Taking the buffer zones on both sides of scenic rivers as an example, referring to Fig. 5, the calculation results of buffer radius at different slope positions are different. The slope map is derived from DEM using GIS 
raster topographic analysis tools. The influence range of both sides of the river is closely related to the topographic slope. See Figure. 5. The distance between the buffer zone boundary and the hidden danger line $\mathrm{L}$ (the center line of the river) changes with the change of the topographic slope. The larger the slope is $(\theta 1 \geq \theta 2)$, the smaller the radius of the projection buffer is ( $\mathrm{R} 1 \leq \mathrm{R} 2)$. When $\theta 1=\theta 2$, the buffer radius $\mathrm{R} 1=\mathrm{R} 2$. $\mathrm{r}$ value, $\mathrm{r}_{\min } \leq r \leq r_{\max }$.

The basic calculation and analysis methods of the planar hidden danger buffer are as follows: in the two-dimensional map, the buffer radius $r$ calculated by formula (1) is set as a plane geometric region based on the periphery edge point of the area $\mathrm{F}$ and the radius. Taking the natural lake buffer zone of scenic spot as an example, referring to Figure 6, the calculation results of buffer radius are different in different lake boundary. The slope map is derived from DEM by using GIS raster terrain analysis tool. The influence range of the surface is closely related to the terrain slope. See Figure 6. The distance between the buffer boundary and the hidden dangerous surface $F$ changes with the change of the terrain slope. The larger the slope is $(\theta 1 \geq \theta 2)$, the smaller the radius of the projection buffer is $(\mathrm{R} 1 \leq \mathrm{R} 2)$. When $\theta 1=\theta 2$, the buffer radius $\mathrm{R} 1=\mathrm{R} 2$. $\mathrm{r}$ value, $\mathrm{r}_{\min } \leq r \leq r_{\max }$.
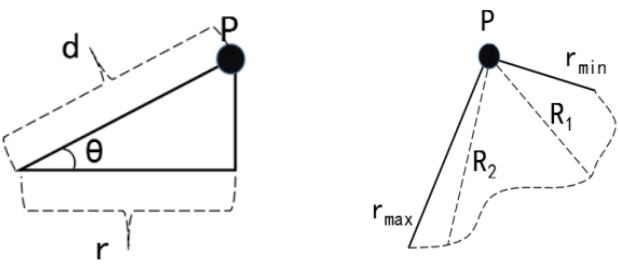

Figure 3. Schematic

Figure 4. Schematic

diagram of buffer radius diagram of potential calculation hazard impact range
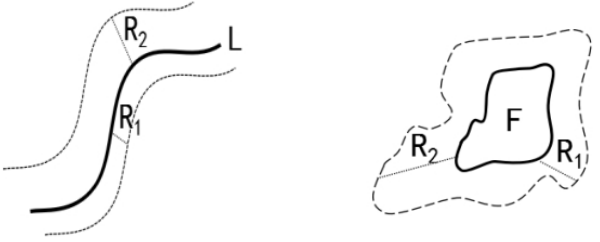

Figure 5. Schematic diagram of potential Figure 6. Diagram of

\section{Hidden Hazard}

hazard impact range

Influencing Range real-time information collection and analysis and processing functions are realized.

In the GIS spatial analysis server of scenic spots, the data of point, line and plane buffer zones are superimposed after the analysis of point, line and plane buffer zones, and the comprehensive hidden danger analysis data and scenic spot security hidden danger map are formed. The hidden danger information after spatial analysis is incorporated into the tourist location safety knowledge base, and compared with the historical hidden danger information in the location safety knowledge base, the temporal and spatial variation rules of the newly added hidden danger characteristics are discovered. Location safety knowledge base is used to store and provide real-time initial values of hidden danger analysis parameters. The data of buffer graphics and buffer minimum radius, maximum radius, neighborhood radius increment, rainfall, water depth increment, visitor location distribution and location flow, hidden danger grade and undetermined coefficient of buffer neighborhood radius increment formula are processed.At the same time, it is used to compare the historical information of buffer parameters and location security knowledge base after real-time acquisition and analysis.It is used to compare and analyze the radius of hidden danger buffer and the characteristics of early warning level.Grammar matching technology is used to record the successful matching information and provide the optimal hidden danger parameters for real-time hidden danger information acquisition and spatial analysis. Location safety knowledge base facilitates the expansion of hidden danger types in scenic spots, thus avoiding repeated collection of unchanged hidden danger objects in scenic spots and reducing unnecessary waste of human and financial resources.

Finally, the new map elements layer is sent to the handheld terminal through the geographic information system network service software, such as Arcgis Server, to guide the collection of potential security hazards information in scenic spots.

\section{REAL-TIME ACQUISITION AND ANALYSIS SYSTEM OF POTENTIAL POSITION SAFETY HAZARD INFORMATION}

\subsection{Module Composition of Information Acquisition System for Hidden Danger of Location Safety}

A real-time acquisition and analysis system module for potential location safety hazard information of intelligent scenic spot tourists in the Internet of Things environment is shown in figure 7. The system includes handheld terminal S1, scenic spot communication base station S2, scenic spot mobile gateway server S3, scenic spot central service network S4, depth sensor $\mathrm{S} 5$, rainfall sensor $\mathrm{S} 6$, video monitor $\mathrm{S} 7$.

Among them, the handheld terminal S1 includes: the handheld device pad and smartphone. Handheld terminals communicate with scenic area central service network S4 through scenic area communication base station S2, scenic area mobile gateway server S3. The application program is mainly used to collect/update the identified or unmarked security data in the system. The handheld terminal not only has the function of location, but also has the function of outdoor query, mapping and geocoding.

Scenic area communication base station S2 refers to a radio transceiver which transmits information bidirectionally between 
the handheld terminal S1 and the mobile gateway server S3 in the scenic area wireless network coverage area for the realization of communication function. Handheld terminal equipment connects the nearest communication base station in the scenic area through WIFI and other channels, and communicates with the mobile gateway server in the scenic area through the base station. Then the hidden danger data collected by the handheld terminal $\mathrm{S} 2$ and spatial analysis requests are transmitted to S4, and the updated data of the system is also transmitted back to $\mathrm{S} 1$.

The Scenic Area Mobile Gateway Server S3 is an inter-network connector and protocol converter, which is used to connect the Scenic Area Communication Base Station S2 and the Scenic Area Central Service Network S4, to realize traffic control, security protection and business isolation, and to provide a safe and efficient access environment for the real-time collection and analysis system of potential safety hazards information for intelligent scenic area tourists.

The scenic area central service network S4 includes the following system parts: Web server S41, GIS spatial database S42, spatial analysis server S43, location security knowledge base S44 and scenic area central local area network S45.

Web server S41, which is used to collect HTTP requests, request storage and spatial analysis operations from a handheld terminal to a Web server.It is also used for comparing and spatial analysis of information sent by handheld terminals via location security knowledge base through spatial analysis server S43 and location security knowledge base S44 when handheld terminal applications send HTTP requests to Web servers. The HTTP response of the Web server is fed back to the handheld terminal to display and guide the collection of security information correctly.

GIS spatial database S42 is used for real-time storage of collected tourist safety data and hidden danger information buffer analysis, overlay analysis results, and realize real-time synchronous storage. The spatial database managed by SDE is used to check the change of topological knowledge in real time according to the topological relationship, so that the spatial data updating can satisfy the consistency and integrity of the topological relationship in the hidden elements layer of scenic spots.

Spatial analysis server S43, which uses real-time spatial analysis component of GIS, provides real-time online spatial analysis service by service pool instance. Specifically, it is used to read location hidden danger data from GIS spatial database S42, and to automatically analyze the buffer area of point, line and surface of hidden danger data, as well as the realization of overlay analysis function.

The scenic area central LAN S45 is used to connect Web server S41, GIS spatial database S42, spatial analysis server S43 and location security knowledge base S44.

Water depth sensor S5 and rainfall sensor S6 are used to obtain real-time water depth and rainfall data, including hourly water depth and daily rainfall data.

Video monitor S7 is used to obtain the density of tourist activity area and tourist flow data of tour routes.

\subsection{Construction of Location Security Knowledge Base}

Location Safety Knowledge Base S44 uses SDE engine of spatial database and relational database to store queries, including index pointer section S441, basic information section S442, knowledge output module S443 and knowledge comparison module S444. See Figure 8.

The index pointer section S441 includes attribute index, spatial index and database pointer. Through the index and pointer part, the relevant hidden danger history information can be obtained only by inputting the information keywords that need to be searched in the index section.

The basic information section is S442, and the hidden danger parameters formed after analysis are stored in the basic information section of the knowledge base. The hidden danger analysis knowledge in the security knowledge base is coded according to different hidden danger types, stored in the SQL Server database, and modified and deleted in the database management system.

The knowledge output module S443 is used to automatically extract the generative rules containing the radius of neighborhood buffer with different hidden dangers affecting the spatial range from the spatial field, attribute field and time field of the knowledge base by writing database scripts and combining with Structured Query Language (SQL). The rule knowledge and analysis result graph are returned to the handheld terminal through HTTP response of Web server to guide the collection and analysis of security hidden danger information.

The knowledge comparison module S444 compares and processes the information sent by the handheld terminal through the location security knowledge base, and feeds back to the handheld terminal. The inaccuracy of buffer radius found by comparing the location safety knowledge base of scenic spots can be checked by scenic area managers, and the knowledge base can be updated and redistributed in the system.

A real-time acquisition and analysis system for potential location safety hazard information of intelligent scenic spots based on Internet of Things provides a set of real-time data acquisition and analysis system based on spatial database and spatial analysis server, which significantly reduces the workload of data acquisition and collation in the later period, and improves the speed of updating potential location safety hazard information of scenic spots.

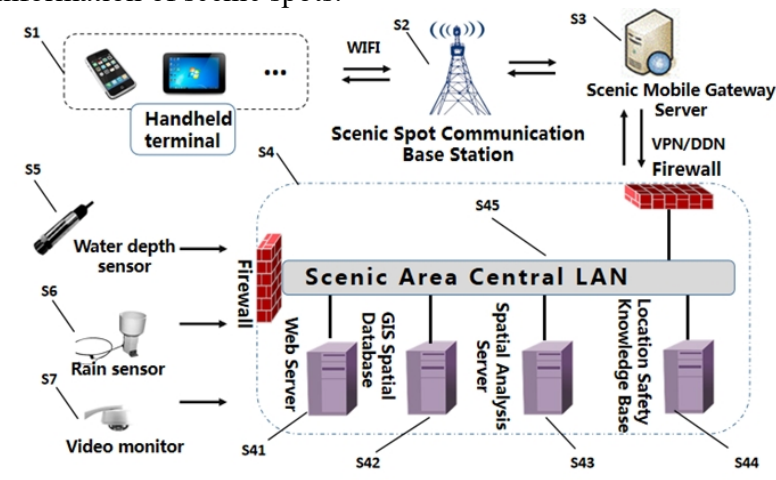

Figure 7. Composition of information acquisition system module for location security hazards 

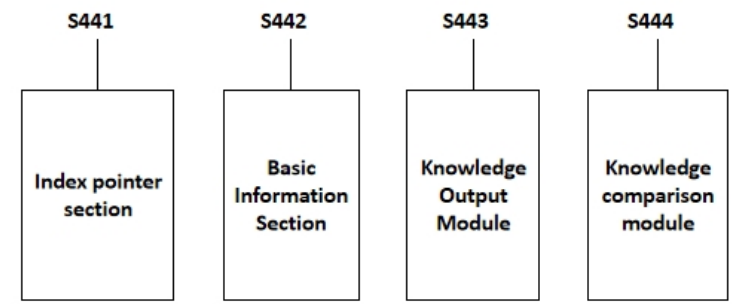

Figure 8. Composition of Location Security Knowledge Base

\section{CONCLUSION}

This paper presents a method and system for real-time collection and analysis of potential location safety hazards of tourists in intelligent scenic spots under the environment of Internet of Things.Based on the image data, the hidden dangers of safety points, lines and areas that have been found or potential are determined. Taking into account the topography, precipitation, location distribution of tourists and location flow data statistics, the range of hidden danger areas that change dynamically with the month and the optimized geographical location of hidden danger areas which are closer to the actual situation are obtained. This method has real-time performance guided by knowledge.Compared with Baidu Map, Gaode Map and other map services, this method can provide more detailed location landmarks and hidden danger information of scenic spots, improve the accuracy of collection and analysis of hidden danger information of scenic spots, and meet the real-time needs of hidden danger prediction and early warning.

Moreover, the creation of intelligent scenic spot location safety knowledge base realizes real-time spatial analysis and facilitates the expansion of scenic spot hidden danger types. Combining location knowledge base, it can improve the efficiency of analysis of hidden danger influence range, avoid repeated collection of unchanged hidden danger objects in scenic spots, and reduce unnecessary waste of manpower and financial resources. This paper provides a real-time data acquisition and analysis system based on spatial database and spatial analysis server, which significantly reduces the workload of data acquisition and collation in the later stage, and improves the speed of updating scenic spot security information.

\section{ACKNOWLEDGMENTS}

This work was supported by the Guilin Scientific Research and Technology Development Plan(20190601;2016012601),Guilin Guilin University of Technology Ph.D Scientific research initial funding(002401003316);Chongqing basic science and advanced technology research(cstc2015jcyjBX0023).Special thanks to the College of Geomatics and Geoinformation, Guilin University of Technology for the support of our work.

\section{REFERENCES}

Chen Rubo., Fu Xiaodong., 2013. Revision of Communication Infrastructure Planning Standards under the Requirement of Smart City Construction. Planner, 29 (6), 62-65.

Dang Anrong., Zhang Danming., Chen Yang., 2011. Study on the Connotation and General Framework of Wisdom Scenic Spots. Chinese Gardens, 27 (9), 15-21.
Hanlin., 2014 Research on Information Product Innovation of Intelligent Scenic Spots Based on Experience Economy. Tourism Forum, 7 (6), 70-73.

Hua Guojun., Liu Xing., Zhou Jianglian.,2009. Brief discussion on tourism safety problems and Countermeasures. Chinese and foreign entrepreneurs, 9(X), 25-26.

Huang Jianfeng,. 2015. Research on the connotation system and construction strategy of intelligent scenic spot based on technology acceptance perspective. Research on science and technology management, (11), 164-168.

Jia Yigang., 2010. Application of Internet of Things in Environmental Monitoring and Early Warning. Shanghai Construction Science and Technology, (06), 65-67.

Shao Zhenfeng., Zhang Xiaoping., Ma Jun., 2010. Jiuzhaigou Intelligent Scenic Spot Management Based on Internet of Things. Geographic Information World, (05), 12-16.

Wang Qiuju., Liu Yu., 2014. Forewarning of tourist flow in scenic spots based on network attention: research framework and empirical analysis--Take National Swimming Center Water Cube as an example. Tourism Forum, 7 (5), 9-15.

Wang Xia., Zhen Feng., Wu Xiaogen., 2015. Evaluation System and Empirical Analysis of Intelligent Scenic Spots Based on Visitors' Perspective: Taking Qinhuai Scenic Belt of Nanjing Confucius Temple as an Example. Progress in Geographic Science, 34 (4), 448-456.

Wang Xianfeng., Ye Xiaoyong., Yang Dan., Wang Liang., 2013. A hand-held data acquisition and storage device. China: ZL201220397011.4, 2013-02-13.

You Weibin., Tan Dehua., Ji Zhirong., 2014. Construction and application of ecological security early warning system for double heritage sites in the world: Taking Wuyishan Scenic Spot as an example. Journal of Applied Ecology, 25 (5), 14551467. 